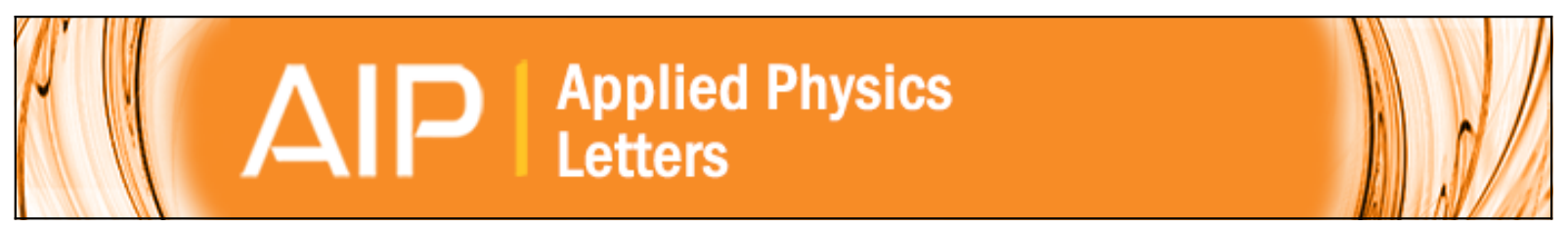

The dielectric breakdown limit of silicone dielectric elastomer actuators

Davide Gatti, Henry Haus, Marc Matysek, Bettina Frohnapfel, Cameron Tropea, and Helmut F. Schlaak

Citation: Applied Physics Letters 104, 052905 (2014); doi: 10.1063/1.4863816

View online: http://dx.doi.org/10.1063/1.4863816

View Table of Contents: http://scitation.aip.org/content/aip/journal/apl/104/5?ver=pdfcov

Published by the AIP Publishing

AlPP Re-register for Table of Content Alerts

Create a profile. 困 Sign up today! 


\title{
The dielectric breakdown limit of silicone dielectric elastomer actuators
}

\author{
Davide Gatti, ${ }^{1,2, a)}$ Henry Haus, ${ }^{2}$ Marc Matysek, ${ }^{2}$ Bettina Frohnapfel, ${ }^{3}$ Cameron Tropea, ${ }^{1}$ \\ and Helmut F. Schlaak ${ }^{2}$ \\ ${ }^{1}$ Center of Smart Interfaces, TU Darmstadt, Alarich-Weiß-Straße 10, 64287 Darmstadt, Germany \\ ${ }^{2}$ Institute of Electromechanical Design, TU Darmstadt, Merckstraße 25, 64287 Darmstadt, Germany \\ ${ }^{3}$ Institute of Fluid Mechanics, Karlsruhe Institute of Technology, Kaiserstraße 10, 76131 Karlsruhe, Germany
}

(Received 23 November 2013; accepted 15 January 2014; published online 4 February 2014)

\begin{abstract}
Soft silicone elastomers are used in a generation of dielectric elastomer actuators (DEAs) with improved actuation speed and durability compared to the commonly used, highly viscoelastic polyacrylate $3 \mathrm{M} \mathrm{VHB}^{\mathrm{TM}}$ films. The maximum voltage-induced stretch of DEAs is ultimately limited by their dielectric breakdown field strength. We measure the dependence of dielectric breakdown field strength on thickness and stretch for a silicone elastomer, when voltage-induced deformation is prevented. The experimental results are combined with an analytic model of equi-biaxial actuation to show that accounting for variable dielectric field strength results in different values of optimal pre-stretch and thickness that maximize the DEA actuation. () 2014 AIP Publishing LLC. [http://dx.doi.org/10.1063/1.4863816]
\end{abstract}

Dielectric elastomers, thanks to their properties of being highly deformable and good electric insulators, have been employed to produce soft, field-induced actuators. ${ }^{1-8}$ The dielectric breakdown strength is the ultimate physical limit that hinders the possibility of actively stretching a dielectric elastomer up to its mechanical failure and, therefore, is of paramount importance in defining the actuator performance bounds. ${ }^{49}$ It does not depend solely on the chemical properties of the material, but also on its physical properties such as mechanical stiffness, ${ }^{10,11}$ thickness, ${ }^{12-17}$ and stretch state, ${ }^{18-20}$ the latter of great interest for dielectric elastomer actuators (DEAs) due to its beneficial effects on actuation. ${ }^{4,9,20,21}$ In literature, the combined effect of thickness and pre-stretch on the breakdown strength of soft elastomers eligible to be used as actuators has been studied mostly for the acrylic elastomer VHB 4910, widely used for early implementations of dielectric elastomer actuators, thanks to its commercial availability as a bonding tape. However, a generation of DEAs capable of large, fast, and reliable actuation is now available and is based on polydimethylsiloxane (PDMS) elastomers with a much lower viscoelasticity than VHB. For this class of elastomers, the combined thickness and stretch dependence on the dielectric field strength has not yet been investigated.

In the present contribution, we study the dielectric breakdown field strength of Elastosil ${ }^{\odot}$ P7670 (Wacker Chemie, Shore A 7) PDMS sheets with 4 different initial thicknesses of $20 \mu \mathrm{m}, 40 \mu \mathrm{m}, 80 \mu \mathrm{m}$, and $200 \mu \mathrm{m}$. The thicker foils are doctor-bladed onto a $90 \mathrm{~mm} \times 1000 \mathrm{~mm}$ glass substrate while the thinner foils are directly spin-coated onto $\varnothing 45 \mathrm{~mm}$ circular copper substrates, according to the process described by Lotz et al. $^{8}$ The $80 \mu \mathrm{m}$ specimens are fabricated as a single-layer film and as two-layer $40 \mu \mathrm{m}$ films, directly superimposed during spin-coating. The thickness of the unstretched samples is precisely measured with a Veeco Dektak 150 + surface profilometer, using a stylus force of $1 \mathrm{mg}$, while for the stretched samples a thickness screw gauge

a)Electronic mail: gatti@csi.tu-darmstadt.de is used. The applied pre-stretches are mostly biaxial, with some additional uniaxial and anisotropic cases, to verify the effect of an eventual anisotropy. The equi-biaxial prestretches are imposed with a multi-clamp, circular pre-stretch jig. A circle of $30 \mathrm{~mm}$ initial diameter $d_{0}$ is drawn on the unstretched specimen and its deformation is used to measure the pre-stretch ratio, defined as $\lambda_{b i}=d_{1} / d_{0}$, where $d_{1}$ is the diameter of the circle after pre-stretch. A four-clamp rectangular pre-stretch jig is used to uniaxially and anisotropically stretch rectangular samples of $90 \mathrm{~mm} \times 80 \mathrm{~mm}$. A $50 \mathrm{~mm} \times$ $50 \mathrm{~mm}$ square is painted onto the PDMS unstretched film to verify homogeneity and amount of pre-stretch. In this case, $\lambda_{x}=x_{1} / x_{0}$ and $\lambda_{y}=y_{1} / y_{0}$ are the pre-stretches in $x$ - (width) and $y$ - (length) planar direction, respectively. In order to compare the amount of pre-stretch of specimens stretched with different stretching techniques, an equivalent stretch ratio $\lambda$ is introduced, defined as the square root of the area stretch. If we assume the material to be incompressible, the equivalent stretch for equi-biaxial state is simply $\lambda=\lambda_{b i}$ while for the anisotropic case $\lambda=\sqrt{\lambda_{x} \lambda_{y}}$. Once stretched, the PDMS films are placed on copper plates, cleaned with isopropyl alcohol, to which they firmly stick. The plate constrains active displacement during the measurements and avoids crack propagation after breakdown.

Our experiments are aimed at measuring the breakdown voltage strength by limiting the active thinning of the dielectric, which can become extreme after electromechanical instabilities, documented by Stark and Garton, ${ }^{22}$ and can lead to premature failure. ${ }^{23}$ The dielectric-breakdown test stand is sketched in Fig. 1. The copper plate acts as a bottom electrode, while the top electrode consists of a copper cylinder of $6 \mathrm{~mm}$ diameter, lowered onto the specimen from the top. The cylinder is polished and has rounded edges in order to reduce field inhomogeneities due to the presences of tips that cause charge accumulation and local deformation. The experiment consists of increasing the voltage (in-house $0-15 \mathrm{kV}$ DC voltage amplifier) stepwise from a safe low limit until the breakdown voltage is reached. The voltage is held constant for $20 \mathrm{~s}$ before increasing in steps of $100 \mathrm{~V}$. 


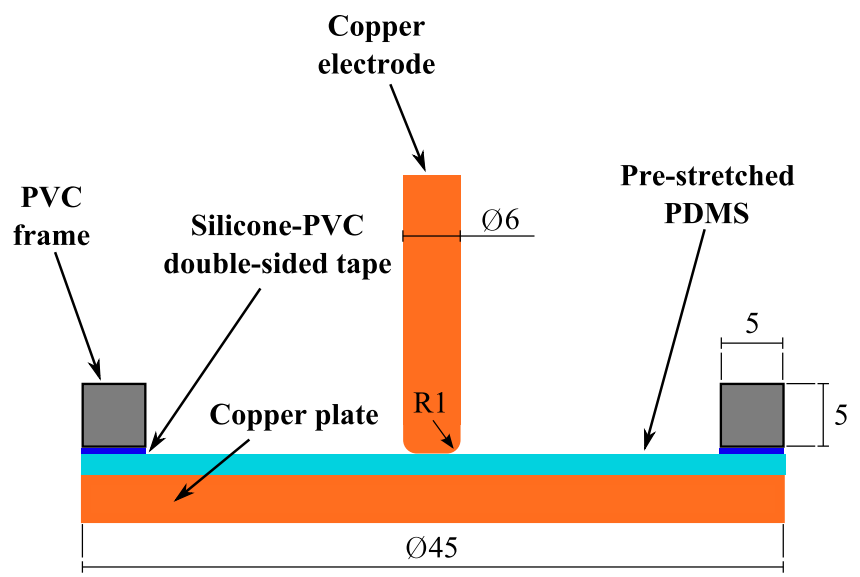

FIG. 1. Schematic of the test stand used for dielectric breakdown field strength. Dimensions in $\mathrm{mm}$.

We define the breakdown as the moment when a sudden and steep increase in leakage current occurs and set the breakdown voltage to the previous voltage level. The nominal dielectric breakdown field strength is then defined as the breakdown voltage $\Phi_{B}$ divided by the measured height $h$ after pre-stretch

$$
E_{B}=\Phi_{B} / h
$$

The results (Figs. 2(a)-2(c)) show that the breakdown field strength of the Elastosil P7670 PDMS elastomer can be significantly enhanced either by decreasing its thickness (Fig. 2(a)) or by increasing the pre-stretch ratio (Fig. 2(b)). A three-fold change in $E_{B}$ has been observed over the investigated parameter range of $h$ and $\lambda$. The breakdown field strength exhibits a clear trend for samples with the same initial thickness $H$, which implies that the dielectric breakdown field strength depends on both thickness and stretch simultaneously, as found by Huang et al. ${ }^{18}$ for the VHB 4910 polyacrylate elastomer. All the data at different thicknesses $h$ and stretch ratios $\lambda$ collapse onto a single line (Fig. 2(c)) if the empirical relation $E_{b}=C h^{\alpha} \lambda^{\beta}$, proposed by Huang et al., ${ }^{18}$ is adopted. The empirical coefficients $\alpha$ and $\beta$ are found by a least-square fit of the entire experimental dataset and lead to the following relation for the dielectric breakdown strength of the Elastosil P7670 silicone elastomer:

$$
E_{B}=147 h^{-0.23 \pm 0.02} \lambda^{0.77 \pm 0.03},
$$

where $h$ is expressed in $\mu \mathrm{m}$ and $E_{B}$ in $\mathrm{V} / \mu \mathrm{m}$, and the confidence level is 95\%. This empirical fit is shown in Fig. 2(c) as a straight line.

The initial thickness $H=\lambda h$ can be used instead of the thickness after pre-stretch $h$ in Eq. (2), leading to the correlation $E_{B}=147 H^{-0.23 \pm 0.02} \lambda^{1.77 \pm 0.03}$. This relation indicates that, as far as an increase in dielectric breakdown field strength is concerned, a higher stretch ratio is more effective than the use of an initially thinner elastomer film.

We investigated whether field inhomogeneities are present that may bias our measurement of the dielectric field strength, using finite element modeling of the experimental setup with the software CST Studio Suite. The PDMS layer is modeled as a dielectric with constant relative permittivity
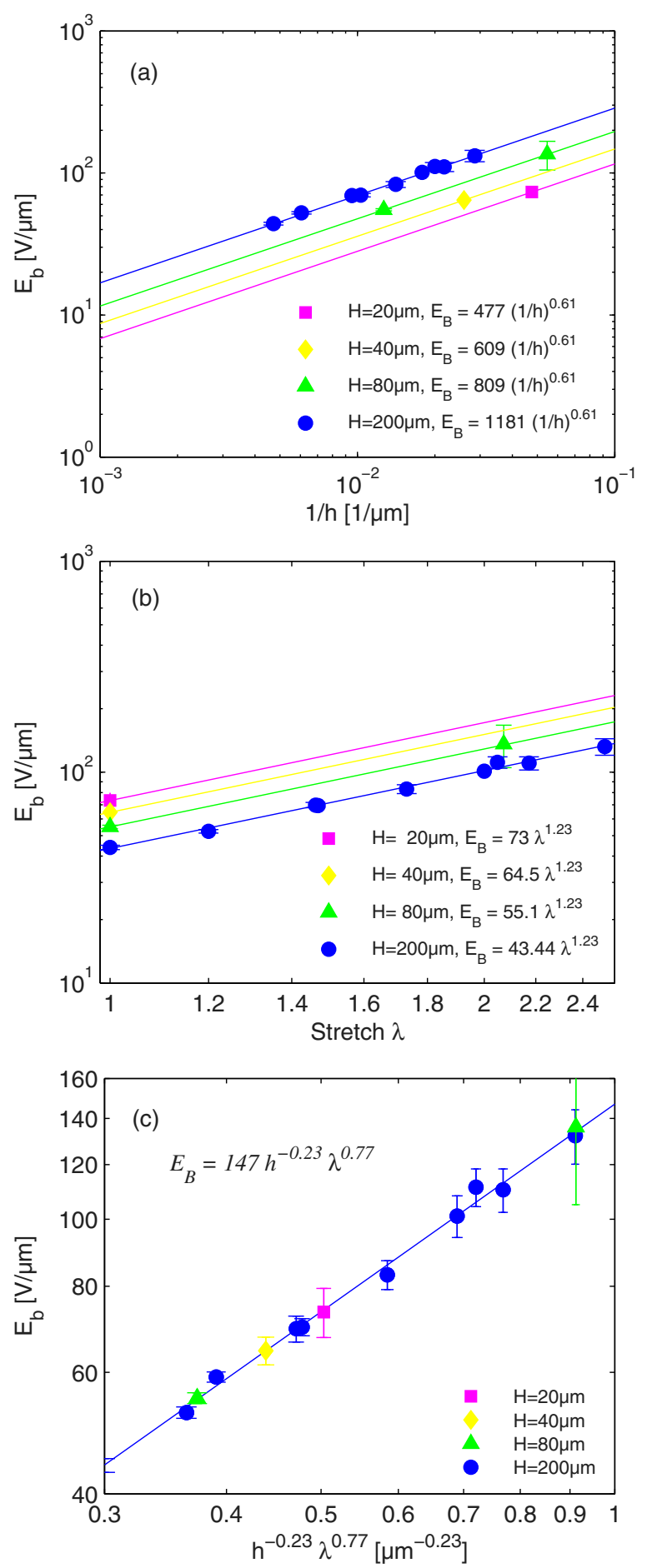

FIG. 2. (a) Dielectric breakdown field strength $E_{B}$ of the silicone elastomer Elastosil P7670 as a function of the thickness $h$ after pre-stretch. (b) Dielectric breakdown field strength $E_{B}$ of the silicone elastomer Elastosil P7670 against the equivalent stretch $\lambda$. (c) Dielectric breakdown field strength $E_{B}$ of the silicone elastomer Elastosil P7670 against the empirically found scaling parameter $h^{-0.23} \lambda^{0.77}$. When multiple lines are present, their slope is obtained from a linear least squares fit to the data for thickness $H=200 \mu \mathrm{m}$. Each line is offset vertically to pass through the data point corresponding to the respective thickness.

$\varepsilon_{r}=3$ and the electrodes are modeled as ideal conductors, while all dimensions are equal to the physical setup (Fig. 1). Figure 3 shows the magnitude of the electric field $E$ along a diameter on the top surface of the dielectric for silicone layers of thickness ranging from 20 to $200 \mu \mathrm{m}$. In the vicinity of the top electrode, edge $E$ presents two local spikes. Here, 


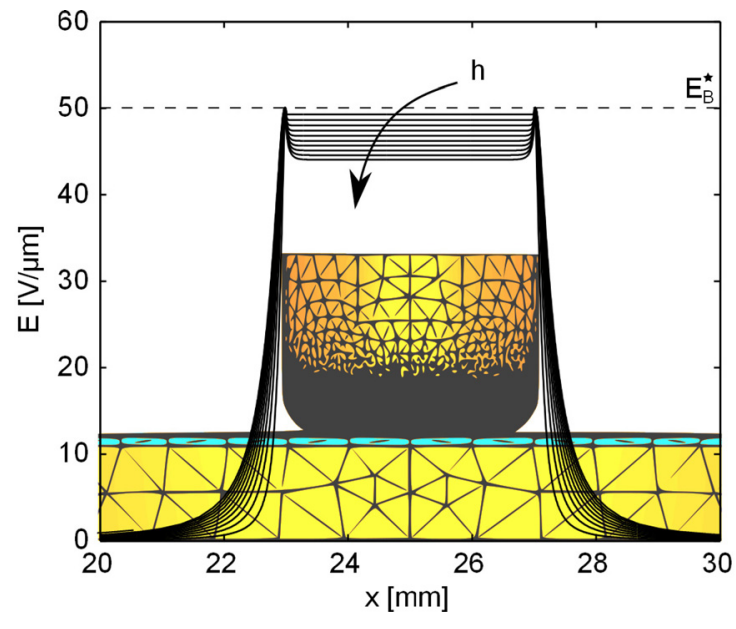

FIG. 3. Magnitude of the electric field $E$ in $V / \mu m$ along a diameter on the top face of the silicone membrane for various thicknesses $h$ of the dielectric; a section of the simulated setup and its numerical discretization is also shown in the background. Two spikes in the electric field strength are present in the vicinity of the top electrode edge, and their amplitude increases with the thickness of the elastomer if the maximum $E$ is kept constant for all simulations.

the intrinsic dielectric breakdown limit $E_{B}^{*}$ (assumed to be $50 \mathrm{~V} / \mu \mathrm{m}$ in Fig. 3) is reached first and is higher than the dielectric breakdown field $E_{B}$ estimated with Eq. (1) at the center of the membrane. The relative difference between $E_{B}^{*}$ and $E_{B}$ increases with the dielectric thickness, possibly leading to an apparent thickness-dependent dielectric field strength. Figure 4 shows the trends of the apparent dielectric breakdown field strength against the thickness for unstretched silicone membranes; both experimental and numerical data points are plotted. In simulations, the apparent dielectric field strength is defined as the magnitude of the electric field in the central part of the membrane when the constant intrinsic breakdown field of $E_{B}^{*}=50 \mathrm{~V} / \mu \mathrm{m}$ is reached in proximity to the electrode edge. We observe that the boundary effects cause a mild thickness-dependence of the dielectric field strength, which is much steeper for experimental data. This means that the particular choice of

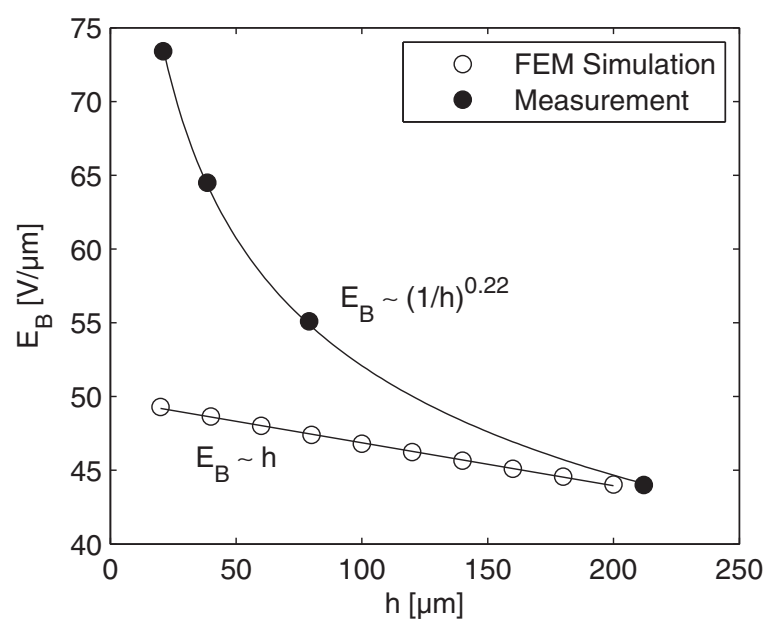

FIG. 4. Trends of the apparent dielectric breakdown field strength against the dielectric thickness. Empty circles: data from a finite element simulation where an intrinsic breakdown $E_{B}^{*}=50$ is assumed; filled circles: experimental data for unstretched $(\lambda=1)$ Elastosil P7670 silicone membranes. electrode shape only marginally affects the measured breakdown strength or the estimated dependence on the thickness.

An important implication when considering or accurately measuring the pre-stretch and thickness dependency of the dielectric breakdown field strength can be highlighted by a simple actuation scenario that can be analytically treated. A circular membrane of dielectric elastomer is equi-biaxially pre-stretched and actuated under constant-force boundary condition. Under the additional hypothesis of incompressibility, the governing equation of DEA is as follows:

$$
\epsilon^{2} \lambda_{\text {act }}^{4} \frac{\Phi^{2}}{h^{2}}=f\left(\lambda \lambda_{a c t}\right)-f(\lambda) \lambda_{\text {act }},
$$

where $\lambda_{\text {act }}$ is the equi-biaxial active stretch defined as $d_{a c t} / d_{1}$ and $f=\mathrm{d} W / \mathrm{d} \lambda$ is the derivative of the strain energy function $W$. We chose to derive the strain energy function with the Gent model $^{24}$ by fitting high-precision uni-, bi-axial, and pure-shear tensile tests. The Gent strain energy function yields high precision in fitting all three experimental tensile tests, compared to other available models. For equi-biaxial actuation the Gent strain energy function takes the following form:

$$
W=-\frac{\mu J_{m}}{2} \ln \left(1-\frac{2 \lambda^{2}-1 / \lambda^{2}-3}{J_{m}}\right),
$$

where $\mu$ is the shear modulus and $J_{m}$ is a constant related to the limiting stretch.

The voltage - active stretch curve for an Elastosil P7670 dielectric elastomer actuator under the aforementioned actuation condition is shown in Fig. 5, assuming a constant dielectric field strength of $E_{B}=70 \mathrm{~V} / \mu \mathrm{m}$, and in Fig. 6, allowing a variable dielectric field strength according to the proposed empirical law. The failure mode of the dielectric elastomer actuator is represented with symbols. Green triangles mark the onset of a pull-in instability, whose consequent large deformation at constant voltage might thin the dielectric beyond its dielectric breakdown strength. Red dots mark

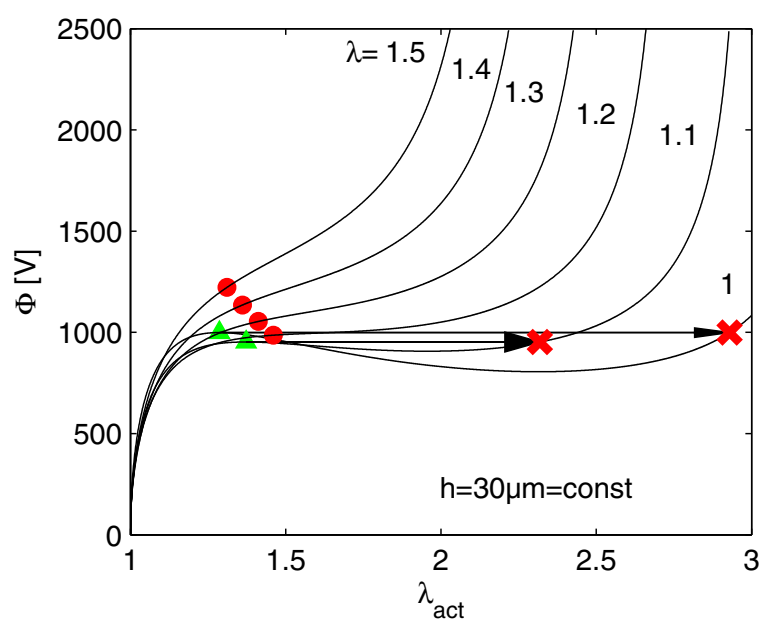

FIG. 5. Voltage $\Phi$ against actuation stretch $\lambda_{\text {act }}$ assuming a constant dielectric breakdown field strength of $70 \mathrm{~V} / \mu \mathrm{m}$. Red circles: failure due to dielectric breakdown; Green triangles: pull-in instability; Red cross: failure due to dielectric breakdown during a pull-in instability. (a) thickness $h$ is kept constant after pre-stretch; (b) thickness $H$ is kept constant before pre-stretch. 

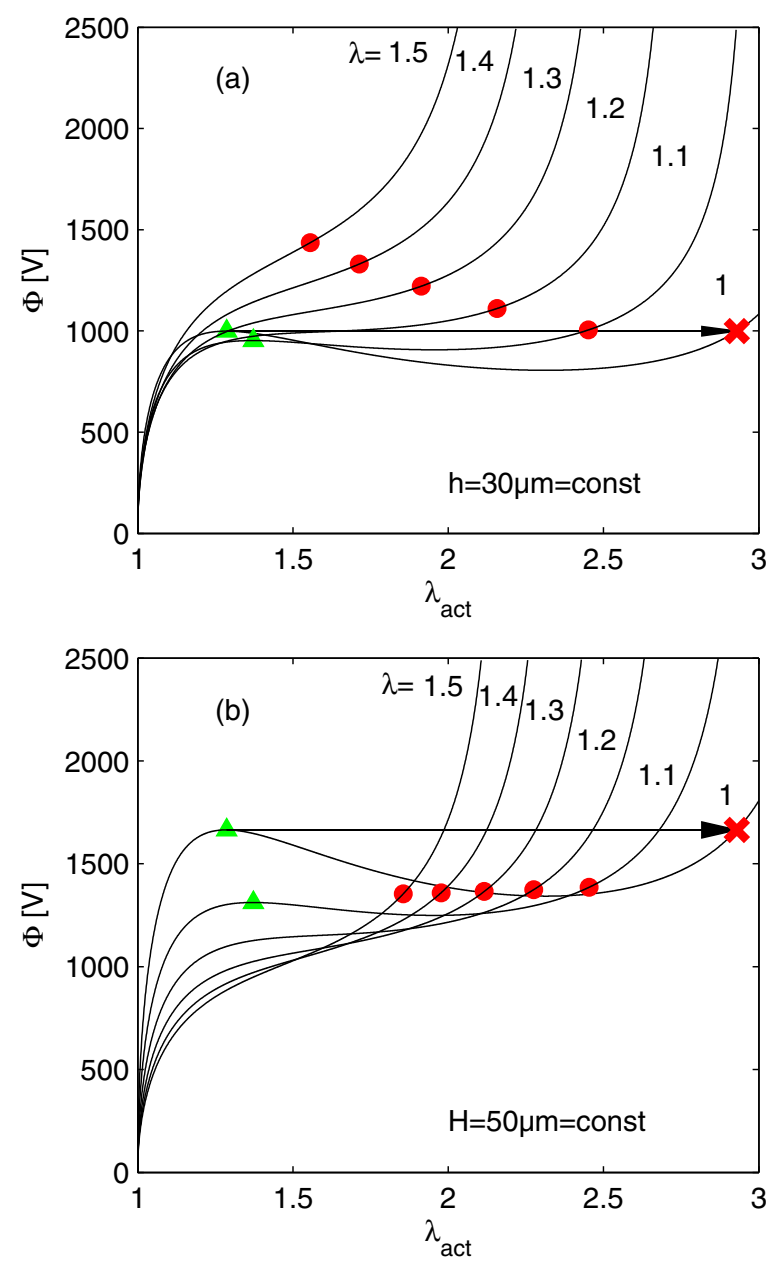

FIG. 6. Voltage $\Phi$ against actuation stretch $\lambda_{\text {act }}$, assuming a variable dielectric breakdown field strength according to Eq. (2). (a) Thickness after prestretch $h$ has been kept constant. (b) Thickness before pre-stretch $H$ has been kept constant. Symbols as in Fig. 5.

the failure due to dielectric breakdown during a stable condition, i.e., when $\mathrm{d} V / \mathrm{d} \lambda_{\text {act }}>0$. We again emphasize the fact that in this framework the only failure mode is dielectric breakdown failure. The electromechanical pull-in instabilities do not directly imply actuator failure, which actually only occurs if the thinning due to the active stretch is so extreme that the breakdown field strength limit is then reached (red crosses).

When a constant dielectric breakdown field strength is assumed (Fig. 5), maximum active stretches of only 1.3 are reached, the only failure mechanism is the dielectric breakdown and the actuator never survives pull-in instabilities. If we account for a variable electric dielectric breakdown field (Fig. 6) the scenario is completely different. The stretch state of the elastomer, i.e., the sum of its pre-stretch $\lambda$ and active stretch $\lambda_{\text {act }}$, might increase the dielectric breakdown field strength and allow the actuator to survive a pull-in instability and reach very large field-induced deformations. Therefore it is not always necessary to pre-stretch until the elimination of the pull-in instability, as suggested by Akbari et al. ${ }^{9}$ This probably led in the past to the misunderstanding that the pull-in instability implies actuator failure.

In Fig. 6, the scenarios with constant thickness $h$ after prestretch (a) and $H$ before pre-stretch (b) are also plotted. The differences in maximum actuation stretch for the two cases at constant $h$ and $H$ is less evident than the prediction by Akbari et al., ${ }^{9}$ if the variable $E_{B}$ is taken into account, because of the higher sensitivity of $E_{B}$ to an increase of pre-stretch than to a decrease of thickness (Eq. (2)). This may influence the design process of DEAs, since silicone membranes with higher initial thickness and pre-stretch ratio may be preferred to thinner and slightly pre-stretched ones. Other actuation scenarios than the bi-axial treated here, like anisotropically pre-stretched membranes, with high pre-stretches in one direction and very low pre-stretches in the perpendicular direction, may take advantage of a significantly higher $E_{B}$ while keeping the capability of high deformations in the low pre-stretch direction.

In conclusion we have experimentally found that the breakdown field strength of a silicone elastomer film used in dielectric elastomer actuators is enhanced by reducing its thickness or increasing the mechanical stretch. We propose the empirical relation $E_{B}=147 h^{-0.23 \pm 0.02} \lambda^{0.77 \pm 0.03}$, similar to the one found by Huang et al. ${ }^{18}$ for a polyacrylate-based elastomer, to describe how the dielectric field strength depends on the thickness and stretch over the investigated range. Once this dependency is accounted for, much larger DEA active stretches are predicted, since dielectric breakdown is the only limit that hinders the possibility to achieve indefinitely large field-induced deformation. As a consequence, the optimal combination of stretch and thickness for a given application may change and then the need of eliminating the pullinstability, as suggested by Akbari et al., ${ }^{9}$ may not always be necessary if dielectric breakdown is considered as a real limit of actuation performance instead of only the pull-in instability.

D.G. is grateful to the Fritz and Margot Faudi Foundation for supporting his research period at TU Darmstadt.

${ }^{1}$ R. Pelrine, R. Kornbluh, Q. Pei, and J. Joseph, Science 287, 836 (2000).

${ }^{2}$ A. O'Halloran, F. O'Malley, and P. McHugh, J. Appl. Phys. 104, 071101 (2008).

${ }^{3}$ P. Brochu and Q. Pei, Macromol. Rapid Commun. 31, 10 (2010).

${ }^{4}$ Z. Suo, Acta Mech. Solida Sin. 23, 549 (2010).

${ }^{5}$ C. Keplinger, T. Li, R. Baumgartner, Z. Suo, and S. Bauer, Soft Matter 8, 285 (2012).

${ }^{6}$ S. J. A. Koh, T. Li, J. Zhou, X. Zhao, W. Hong, J. Zhu, and Z. Suo, J. Polym. Sci., Part B: Polym. Phys. 49, 504 (2011).

${ }^{7}$ X. Zhao and Z. Suo, Phys. Rev. Lett. 104, 178302 (2010).

${ }^{8}$ P. Lotz, M. Matysek, and H. F. Schlaak, IEEE/ASME Trans. Mechatron. 16, 58 (2011).

${ }^{9}$ S. Akbari, S. Rosset, and H. R. Shea, Appl. Phys. Lett. 102, 071906 (2013).

${ }^{10}$ M. Kollosche and G. Kofod, Appl. Phys. Lett. 96, 071904 (2010).

${ }^{11}$ M. Kollosche, H. Stoyanov, H. Ragusch, S. Risse, A. Becker, and G. Kofod, in Proceedings of the 2010 10th IEEE International Conference on Solid Dielectrics (ICSD 2010), Potsdam, Germany, 4-9 July 2010 (IEEE Piscataway, NJ, USA, 2010), pp. 1-4.

${ }^{12}$ A. P. Gerratt and S. Bergbreiter, J. Micromech. Microeng. 23, 067001 (2013).

${ }^{13}$ B. Helgee and P. Bjellheim, IEEE Trans. Electr. Insul. 26, 1147 (1991).

${ }^{14}$ M. Danikas, IEEE Trans. Dielectr. Electr. Insul. 1, 1196 (1994).

${ }^{15}$ V. Agarwal and V. Srivastava, Thin Solid Films 8, 377 (1971).

${ }^{16}$ H. Kim and F. Shi, IEEE Trans. Dielectr. Electr. Insul. 8, 248 (2001).

${ }^{17}$ F. Forlani and N. Minnaja, Phys. Status Solidi B 4, 311 (1964).

${ }^{18}$ J. Huang, S. Shian, R. M. Diebold, Z. Suo, and D. R. Clarke, Appl. Phys. Lett. 101, 122905 (2012).

${ }^{19}$ G. Kofod, P. Sommer-Larsen, R. Kornbluh, and R. Pelrine, J. Intell. Mater. Syst. Struct. 14, 787 (2003).

${ }^{20}$ J.-S. Plante and S. Dubowsky, Int. J. Solids Struct. 43, 7727 (2006).

${ }^{21}$ G. Kofod, J. Phys. D: Appl. Phys. 41, 215405 (2008).

${ }^{22}$ K. H. Stark and C. G. Garton, Nature 176, 1225 (1955).

${ }^{23}$ J. Blok, J. Appl. Phys. 40, 288 (1969).

${ }^{24}$ A. Gent, Rubber Chem. Technol. 69(1), 59 (1996). 